\title{
Perfil epidemiológico de pacientes idosos atendidos em um pronto-socorro de hospital universitário brasileiro
}

\author{
Epidemiological profile of elderly patients treated in the emergency room of a \\ university hospital in Brazil
}

\section{Cacilda Pedrosa de Oliveira ${ }^{1}$, Isadora Marques Guimarães Santos ${ }^{2}$, Andrey Rocha Rocca ${ }^{2}$, Giovani Prediger Dobri², Gabriel Duarte do Nascimento ${ }^{2}$}

\begin{abstract}
Oliveira CP, Santos IMG, Rocca AR, Dobri GB, Nascimento GD. Perfil epidemiológico de pacientes idosos atendidos em um prontosocorro de hospital universitário brasileiro / Epidemiological profile of elderly patients treated in the emergency room of a university hospital in Brazil. Rev Med (São Paulo). 2018 jan.-fev.;97(1):44-50.
\end{abstract}

RESUMO: Este estudo objetiva descrever características demográficas, informações sobre internação e antecedentes médicos, bem como traçar o perfil epidemiológico de idosos atendidos no Pronto-Socorro (PS) do Hospital das Clínicas - UFG (HCUFG). Trata-se de um estudo transversal e descritivo. Através da análise de prontuários, de janeiro de 2015 a julho de 2016 , foram selecionados pacientes com 60 anos ou mais, que permaneceram no PS por mais de 24 horas ou vieram à óbito neste período. Baseado nos prontuários, foram pesquisadas as seguintes variáveis: aspectos sociodemográficos, queixas sintomáticas, causas de internação, complicações durante a internação, antecedentes (comorbidades e medicamentos em uso) e condições de alta. Dos 743 pacientes adultos atendidos no período, $29,87 \%$ eram idosos, totalizando 222 pacientes estudados. A mediana de idade foi 69 anos, sendo a maioria do sexo masculino. As principais queixas referidas no processo de triagem foram náuseas e vômitos. Infecção foi a causa de internação mais frequente e a maioria dos pacientes evoluiu sem complicações. Hipertensão e diabetes mellitus foram as comorbidades mais prevalentes $(65,77 \%$ e $43,24 \%$, respectivamente). Aproximadamente $41,89 \%$ dos pacientes apresentavam polifarmácia. A duração média da permanência no hospital foi 4,9 dias. No período, $18,47 \%$ vieram a óbito e $71,17 \%$ receberam alta com melhora do quadro clínico. Em geral, os resultados do estudo foram compatíveis com a literatura. Espera-se que os dados desse estudo contribuam para o conhecimento nacional sobre pacientes idosos atendidos em serviços médicos de emergência, importante para o desenvolvimento de abordagens clínicas mais direcionadas e resolutivas.

Descritores: Serviços de saúde para idosos; Serviços médicos de emergência; Perfil de saúde; Geriatria.
ABSTRACT: This study aims to describe demographic characteristics, hospitalization information and medical history, as well as to trace the epidemiological profile of elderly patients treated in the Emergency Room (ER) of the Hospital das Clínicas - UFG (HCUFG). It is a cross-sectional and descriptive study. Through the analysis of medical records, from January 2015 to July 2016, patients aged 60 years or more who remained in the ER for over 24 hours or died during this period were selected. Based on the medical records, the following variables were searched: sociodemographic aspects, symptomatic complaints, causes of hospital admission, complications during hospital stay, medical history (comorbidities and medications in use) and discharge conditions. From the 743 adult patients seen in the period, $29.87 \%$ were elderly, totaling 222 patients studied. The median age of the elderly assisted was 69 years old, with the majority being male. The main complaints referred to in the screening process were nausea and vomiting. Infection was the most frequent cause of hospitalization, and most of the patients evolved without complications. Hypertension and diabetes mellitus were the most prevalent comorbidities $(65,77 \%$ e $43,24 \%$, respectively). Approximately $41,89 \%$ of the patients presented polypharmacy. The mean length of hospital stay was 4.9 days. During the period, $18,47 \%$ died and $71.17 \%$ were discharged with improvement of their clinical condition. In general, the results were compatible with the literature. It is expected that this data contribute to national knowledge about elderly patients seen in the emergency medical services, which is important to develop more directed and resolutive clinical approaches.

Keywords: Health services for the aged; Emergency medical services; Health profile, Geriatrics.

1. Hospital das Clínicas da Universidade Federal de Goiás, Goiânia, GO, BR. Médica. E-mail: cpedrosa1@gmail.com, https://orcid.org/00000002-7191-0322.

2. Faculdade de Medicina da Universidade Federal de Goiás, Goiânia, GO, BR. Acadêmicos do $8^{\circ}$ período do Curso de Medicina da UFG. E-mail: ms.isa.marqs@gmail.com, https://orcid.org/0000-0001-7596-9391; andrey.rocca@gmail.com, https://orcid.org/0000-0003-28658018; giovani05@msn.com, https://orcid.org/0000-0003-3508-7305; gabrielmedufg@yahoo.com.br. https://orcid.org/0000-0001-9381-5572. Endereço para correspondência: Isadora Marques Guimarães Santos. Rua 235, Qd. 56, Lt. 10, Número 101, Ed. Iguatemi, Apt. 301. Setor Leste Universitário. Goiânia, GO, BR. 


\section{INTRODUÇÃO}

$\mathrm{m}$ todo o mundo, o número absoluto e relativo
de idosos tem aumentado drasticamente como um reflexo do aumento da expectativa de vida e da queda das taxas de fertilidade. As melhorias socioeconômicas contribuíram para esse processo, aumentando as chances de sobreviver em idades mais jovens e, consequentemente, de chegar à terceira idade ${ }^{1}$. No Brasil, a transição demográfica acentuou-se a partir de $1950^{2}$. De 1960 a 2010, o número de idosos aumentou $600 \%$ e, estima-se que em 2020, correspondam a $14 \%$ da população brasileira ${ }^{3}$.

Essa mudança de padrão demográfico reflete-se de forma importante nas características de adoecimento da população. O envelhecimento biológico caracteriza-se por um acúmulo de danos moleculares e celulares, que podem gerar declínio funcional e aumento da vulnerabilidade e do risco de doenças ${ }^{1}$. De modo geral, prevalecem as doenças crônico-degenerativas em relação às infectoparasitárias. Na população acima de 60 anos, as doenças não transmissíveis já correspondem a mais de $87 \%$ dos agravos de saúde ${ }^{4}$.

Como consequência do exposto, novos padrões de cuidados em saúde devem se estabelecer nos próximos anos $^{4}$. Um dos aspectos a serem avaliados é a utilização dos serviços de emergência pelos idosos. Neste contexto, eles têm características particulares que refletem em maior tempo de hospitalização e chances aumentadas de reinternação, contribuindo para a superlotação dos ProntoSocorros e aumento dos custos de saúde 5 .

$\mathrm{O}$ atendimento de pacientes idosos agudamente fragilizados no PS é um desafio para profissionais que frequentemente não são capacitados para lidar com especificidades desse grupo, como apresentações atípicas de doenças, comorbidades e polifarmárcia, por exemplo ${ }^{6}$. Diante desse cenário, este estudo teve por objetivo descrever as características demográficas e epidemiológicas dos idosos que compareceram ao Pronto-Socorro do Hospital das Clínicas da UFG, de janeiro de 2015 a julho de 2016, visando estabelecer um perfil que auxilie a compreensão desse grupo heterogêneo de pacientes.

\section{MÉTODOS}

\section{Desenho do estudo e seleção de participantes}

Trata-se de um estudo transversal e descritivo, que visou descrever características demográficas, informações sobre internação e antecedentes médicos, bem como traçar, através de análise de prontuários, o perfil epidemiológico dos pacientes com sessenta anos ou mais que chegaram ao Pronto-Socorro de um hospital universitário, no período de 01 de janeiro de 2015 até 30 de julho de 2016.

Os pacientes atendidos na unidade de emergência foram a população alvo do estudo, desde que correspondessem aos seguintes critérios de inclusão: com 60 anos ou mais, atendidos na unidade de emergência, que se mantiveram nesta por mais de 24 horas ou foram à óbito neste período.

Variáveis

Os prontuários dos pacientes foram analisados quanto a: dados sociodemográficos; queixas somáticas; causas de internação; complicações durante o período; histórico de comorbidades; presença de polifarmácia, e condições de alta. Os dados coletados incluem: principais queixas na triagem; período de permanência na unidade de emergência e, se fosse o caso, também em outras unidades do hospital; internação ou não em unidade de terapia intensiva.

Avaliou-se ainda as principais complicações, comorbidades, condições de alta (se mostrou melhora, alta na dependência de cuidados, necessidade de home care ou de internação asilar), e o desfecho de óbito ou não. Foi levantado o número de medicamentos de uso contínuo. Para coleta de dados padronizada, com objetivação de descritores, foram utilizadas as definições apresentadas no Quadro 1.

Quadro 1. Definições conceituais adotadas para coleta de dados padronizada

\begin{tabular}{|c|c|}
\hline Descritor & Definição \\
\hline $\begin{array}{l}\text { Doenças } \\
\text { pulmonares } \\
\text { crônicas }\end{array}$ & $\begin{array}{l}\text { Doenças crônicas que implicam em prejuízo no } \\
\text { funcionamento pulmonar, como asma e doença } \\
\text { pulmonar obstrutiva crônica. }\end{array}$ \\
\hline $\begin{array}{l}\text { Doenças } \\
\text { degenerativas } \\
\text { do SNC }\end{array}$ & $\begin{array}{l}\text { Doenças com morte e degeneração de células do } \\
\text { sistema nervoso central, como esclerose múltipla, } \\
\text { esclerose lateral amiotrófica, esclerose tuberosa, } \\
\text { síndrome de Parkinson e doença de Alzheimer. }\end{array}$ \\
\hline $\begin{array}{l}\text { Doenças } \\
\text { hepáticas }\end{array}$ & $\begin{array}{l}\text { Doenças que atingem o fígado e ocasionam alteração } \\
\text { estrutural e/ou deficiência funcional como cirrose, } \\
\text { hepatites e colangite esclerosante. }\end{array}$ \\
\hline $\begin{array}{l}\text { Doenças } \\
\text { renais }\end{array}$ & $\begin{array}{l}\text { Doenças que atingem os rins, causando alteração } \\
\text { estrutural e/ou deficiência funcional como } \\
\text { nefrolitíase, insuficiência renal e nefropatia diabética. }\end{array}$ \\
\hline $\begin{array}{l}\text { Disfunções } \\
\text { gastrointestinais }\end{array}$ & $\begin{array}{l}\text { Doenças que atingem o trato gastrointestinal e } \\
\text { órgãos acessórios da digestão ocasionando alteração } \\
\text { estrutural e/ou deficiência funcional como doença do } \\
\text { refluxo gastroesofágico. }\end{array}$ \\
\hline $\begin{array}{l}\text { Disfunções } \\
\text { endocrinológicas }\end{array}$ & $\begin{array}{l}\text { Doenças que provocam alterações estruturais } \\
\text { em glândulas endócrinas e/ou função anômala } \\
\text { de secreções hormonais, como diabetes, } \\
\text { hipertireoidismo e síndrome de Cushing. }\end{array}$ \\
\hline $\begin{array}{l}\text { Distúrbios } \\
\text { hidroeletrolíticos }\end{array}$ & $\begin{array}{l}\text { Alterações funcionais decorrentes de níveis anormais } \\
\text { de eletrólitos como hipernatremia, hiponatremia e } \\
\text { hipercalcemia. }\end{array}$ \\
\hline $\begin{array}{l}\text { Complicações } \\
\text { renais }\end{array}$ & $\begin{array}{l}\text { Evento agudo com agravamento de condição clínica } \\
\text { durante evolução de doença renal, como alterações } \\
\text { neurológicas, sangramentos e infecções. }\end{array}$ \\
\hline $\begin{array}{l}\text { Complicações } \\
\text { hepáticas }\end{array}$ & $\begin{array}{l}\text { Evento agudo com agravamento de condição } \\
\text { clínica durante evolução de doença hepática, como } \\
\text { encefalopatia hepática e abscesso hepático. }\end{array}$ \\
\hline $\begin{array}{l}\text { Complicações } \\
\text { neuropsiquiátricas }\end{array}$ & $\begin{array}{l}\text { Agravamento de condição clínica durante evolução } \\
\text { de doença neuropsiquiátrica como ansiedade, quadros } \\
\text { psicóticos e comportamentos parassuicidários e } \\
\text { suicidários. }\end{array}$ \\
\hline $\begin{array}{l}\text { Complicações } \\
\text { de doenças } \\
\text { oncológicas }\end{array}$ & $\begin{array}{l}\text { Agravamento de condição clínica durante evolução } \\
\text { de doença oncológica como hipercalcemia, síndrome } \\
\text { da veia cava superior e obstrução de via aérea. }\end{array}$ \\
\hline $\begin{array}{l}\text { Complicações } \\
\text { gastrointestinais }\end{array}$ & $\begin{array}{l}\text { Agravamento de condição clínica durante evolução } \\
\text { de doença gastrointestinal como hemorragia } \\
\text { digestiva, íleo paralítico e apendicite. }\end{array}$ \\
\hline $\begin{array}{l}\text { Interação } \\
\text { medicamentosa/ } \\
\text { polimedicação }\end{array}$ & $\begin{array}{l}\text { Evento clínico em que os efeitos de um fármaco são } \\
\text { alterados pela presença de outro fármaco, alimento, } \\
\text { bebida ou agente químico ambiental. }\end{array}$ \\
\hline
\end{tabular}




\section{Análise dos dados}

Realizou-se análise descritiva quantitativa e qualitativa das variáveis. Para isso, utilizou-se o software Excel 2013 para tabulação e ActionStat, para análise estatística.

\section{Aspectos éticos}

O projeto foi submetido ao Comitê de Ética em Pesquisa do Hospital das Clínicas da Universidade Federal de Goiás, sendo aprovado na data de 18/02/2016. Foi dispensado por este da utilização de Termo de Consentimento Livre Esclarecido. Assim, todos os aspectos contidos na Resolução do CNS 466/2012 foram respeitados.

\section{RESULTADOS}

Em um universo de 743 pacientes adultos atendidos no período de 17 meses, 29,87\% eram idosos e passaram mais de 24 horas na unidade de Pronto-Socorro. Dessa forma, 222 pacientes foram incluídos no estudo segundo critérios metodológicos definidos.

Dentre os 222 pacientes, a maioria apresentava 69 anos, o que também corresponde à mediana, com o primeiro e o terceiro quartis iguais a 64 e 76 anos, respectivamente. Além disso, $113(50,90 \%)$ eram do sexo masculino e $108(48,65 \%)$ do sexo feminino. Em relação à etnia, 109 (49,10\%) dos atendidos eram autodeclarados pardos, 95 (42,79\%) não informaram, $12(5,41 \%)$ eram brancos, 4 $(1,80 \%)$ eram negros e $1(0,45 \%)$ índio.

Quadro 2. Caracterização da amostra de pacientes idosos atendidos no Pronto-Socorro de um Hospital Universitário brasileiro de 2015 a 2016, segundo idade, sexo e etnia

\begin{tabular}{|c|c|c|}
\hline & Quantidade & $\%$ \\
\hline \multicolumn{3}{|l|}{ Idade } \\
\hline Mediana & 69 & \\
\hline $1^{\circ}$ quartil & 64 & \\
\hline $3^{\circ}$ quartil & 76 & \\
\hline \multicolumn{3}{|l|}{ Sexo } \\
\hline Masculino & 113 & $50,90 \%$ \\
\hline Feminino & 108 & $48,65 \%$ \\
\hline \multicolumn{3}{|l|}{ Etnia } \\
\hline Negro & 4 & $1,80 \%$ \\
\hline Branco & 12 & $5,41 \%$ \\
\hline Pardo & 109 & $49,10 \%$ \\
\hline Índio & 1 & $0,45 \%$ \\
\hline Não dito & 95 & $42,79 \%$ \\
\hline
\end{tabular}

As principais queixas reportadas pelos pacientes na triagem estão mostradas no Quadro 3. Observa-se que $25 \%$ dos pacientes se apresentaram com náuseas e vômitos, $22,07 \%$ com dispneia e 19,82\% com astenia/fraqueza.
Quadro 3. Principais queixas da triagem de pacientes idosos atendidos no Pronto-Socorro em um Hospital Universitário brasileiro de 2015 a 2016

\begin{tabular}{|l|c|c|}
\hline Sintomas & Quantidade & \% \\
\hline Náuseas/vômitos & 56 & $25,23 \%$ \\
\hline Dispneia & 49 & $22,07 \%$ \\
\hline Astenia/fraqueza & 44 & $19,81 \%$ \\
\hline Febre & 35 & $15,77 \%$ \\
\hline Tosse & 33 & $14,86 \%$ \\
\hline Dor Abdominal & 30 & $13,51 \%$ \\
\hline Dor torácica/precordial & 20 & $9,01 \%$ \\
\hline $\begin{array}{l}\text { Queixas TGU (hematúria, disúria } \\
\text { ou alterações do volume urinário, } \\
\text { incontinência ou retenção) }\end{array}$ & 20 & $9,01 \%$ \\
\hline Inapetência/hiporexia & 16 & $7,21 \%$ \\
\hline Confusão mental & 15 & $6,76 \%$ \\
\hline
\end{tabular}

O Quadro 4 descreve as principais causas de internação dos pacientes. Infecção foi a causa mais frequentemente observada $(26,13 \%)$, seguida por complicações de doenças oncológicas $(13,96 \%)$. É importante também notar que complicações renais $(9,91 \%)$ e insuficiência cardíaca $(8,56 \%)$ foram a quarta e a quinta causas mais prevalentes.

Quadro 4. Causas mais frequentes de internação de pacientes idosos atendidos no Pronto-Socorro em um Hospital Universitário brasileiro de 2015 a 2016

\begin{tabular}{|l|c|c|}
\hline Causa & Quantidade & \% \\
\hline Infecção & 58 & $26,13 \%$ \\
\hline \multicolumn{1}{|c|}{ Pulmão } & 32 & $55,17 \%$ \\
\hline \multicolumn{1}{|c|}{ TGU } & 13 & $22,41 \%$ \\
\hline \multicolumn{1}{|c|}{ Outros } & 11 & $18,97 \%$ \\
\hline $\begin{array}{l}\text { Complicações de doenças } \\
\text { oncológicas }\end{array}$ & 2 & $3,45 \%$ \\
\hline Outras & 31 & $13,96 \%$ \\
\hline Complicações renais & 22 & $13,96 \%$ \\
\hline Insuficiência cardíaca & 19 & $8,91 \%$ \\
\hline Complicações hepáticas & 15 & $6,76 \%$ \\
\hline Doença coronariana & 14 & $6,31 \%$ \\
\hline Complicações gastrointestinais & 14 & $6,31 \%$ \\
\hline $\begin{array}{l}\text { Doenças pulmonares crônicas/ } \\
\text { disfunções pulmonares }\end{array}$ & 13 & $5,86 \%$ \\
\hline Abdome agudo & 6 & $2,70 \%$ \\
\hline
\end{tabular}

Durante a internação, conforme apresentado no Quadro 5, a maioria dos pacientes $(61,71 \%)$ não apresentou complicações. No entanto, cerca de 25 pacientes $(11,26 \%)$ 
Oliveira CP, et al. Perfil epidemiológico de pacientes idosos atendidos em um pronto-socorro de hospital.

evoluíram com parada cardiorrespiratória e 17 (7,66\%) adquiriram algum tipo de infecção no período que passaram internados no hospital, conforme registrado nos prontuários.

Quadro 5. Principais complicações durante a internação de pacientes idosos atendidos no Pronto-Socorro em um Hospital Universitário brasileiro de 2015 a 2016

\begin{tabular}{|l|c|c|}
\hline & Quantidade & \% \\
\hline Ausência de complicações & 103 & $61,71 \%$ \\
\hline Parada cardiorrespiratória & 25 & $11,26 \%$ \\
\hline Infecção & 17 & $7,66 \%$ \\
\hline \multicolumn{1}{|c|}{ Sepse } & 6 & $33,33 \%$ \\
\hline Pulmão & 5 & $27,78 \%$ \\
\hline TGU & 4 & $22,22 \%$ \\
\hline Abdome & 2 & $11,11 \%$ \\
\hline Outras & 12 & $5,41 \%$ \\
\hline Complicações renais & 10 & $4,50 \%$ \\
\hline Distúrbios hidroeletrolíticos & 7 & $3,15 \%$ \\
\hline Complicações hepáticas & 5 & $2,25 \%$ \\
\hline $\begin{array}{l}\text { Complicações } \\
\text { neuropsiquiátricas }\end{array}$ & 4 & $1,80 \%$ \\
\hline Delirium & 3 & $1,35 \%$ \\
\hline Acidente vascular encefálico & 1 & $0,45 \%$ \\
\hline Interações medicamentosas & 1 & $0,45 \%$ \\
\hline
\end{tabular}

Os principais sítios de infecção nos pacientes idosos que foram internados pelo setor de emergência estão descritos no Quadro 4. O pulmão foi o principal sítio de infecção presente no momento da internação $(55,17 \%)$, seguido do trato geniturinário $(22,41 \%)$. Esse mesmo padrão ocorre quando são analisadas infecções adquiridas no Pronto-Socorro pelos pacientes que estão internados nesta unidade (sítios de infecções adquiridas como complicações), ilustrado no Quadro 5: pulmão (27,78\%) e trato urinário (22,22\%). Outro dado relevante é que 33,33\% dos pacientes que adquiriram infecção durante a internação apresentaram quadro de sepse.

De acordo com o Quadro 6, a maioria desses idosos tinha hipertensão arterial sistêmica $(65,77 \%)$ e 43,24\% apresentavam diabetes mellitus. Além disso, cerca de 17,56\% dos pacientes tinham histórico de câncer. Já 16,67\% não apresentavam nenhuma comorbidade. Vale salientar que $56,30 \%$ apresentavam pelo menos 3 comorbidades, resultando em um primeiro quartil de 2 e terceiro quartil de 4 comorbidades por paciente. Em adição, observa-se que
$41,89 \%$ dos enfermos tinham polifarmácia (uso de mais de 5 medicamentos).

Quadro 6. Principais comorbidades apresentadas por pacientes idosos atendidos no Pronto-Socorro em um Hospital Universitário brasileiro de 2015 a 2016

\begin{tabular}{|l|c|c|}
\hline & Quantidade & \% do total \\
\hline Hipertensão arterial sistêmica & 146 & $65,77 \%$ \\
\hline Diabetes mellitus & 96 & $43,24 \%$ \\
\hline Câncer & 39 & $17,57 \%$ \\
\hline Sem comorbidades & 37 & $16,67 \%$ \\
\hline Histórico de AVE & 36 & $16,22 \%$ \\
\hline Dislipidemia & 33 & $14,86 \%$ \\
\hline Outros & 33 & $14,86 \%$ \\
\hline Tuberculose & 32 & $14,41 \%$ \\
\hline Cardiopatia & 30 & $13,51 \%$ \\
\hline Doenças renais & 25 & $11,26 \%$ \\
\hline
\end{tabular}

Em relação ao tempo de permanência desses idosos no hospital, observou-se que a maior parte permaneceu 4 dias internados no Pronto-Socorro, sendo 2 dias o primeiro quartil e 6 dias o terceiro quartil. As condições de alta desses enfermos foram: $71,17 \%$ receberam alta com melhora do quadro e $9,46 \%$ com alta dependente de cuidados. $\mathrm{O}$ número de óbitos nesse período foi de $41(18,47 \%)$.

\section{DISCUSSÃO}

O percentual de idosos atendidos $(29,87 \%)$ no PS-HCUFG está acima da prevalência média referida pela literatura ${ }^{8}$. Este resultado reflete uma tendência que deve se acentuar progressivamente, em concordância com a transição demográfica ${ }^{8}$. Além disso, é compatível com as características do local do estudo: um Hospital Universitário terciário, cuja porta de entrada é regulada e direcionada para indivíduos com doenças crônicodegenerativas, que habitualmente acometem os pacientes de faixa etária mais elevada. Em termos demográficos, a mediana de idade (69 anos) e a ligeira predominância do sexo masculino $(50,9 \%)$ é semelhante ao encontrado na literatura consultada ${ }^{2}$.

Ao serem recebidos no PS-HCUFG, estes pacientes passaram por um processo de triagem, onde as principais queixas foram registradas (náuseas e vômitos, dispneia, astenia, febre, tosse, dor abdominal e dor torácica). Até $25 \%$ dos pacientes podem chegar ao PS com queixas inespecíficas, como astenia, dispneia e mal estar. Embora 
estas queixas possam ser vinculadas a um pior prognóstico, tal fato não foi confirmado no estudo comparado? .

Segundo a literatura, dentre as principais queixas dos pacientes idosos que chegam no PS, estão: dispneia, dor torácica, dor abdominal e cólicas, astenia e tontura ${ }^{10}$. Em um estudo realizado nos Estados Unidos em 2013, foram avaliadas as queixas de internação de 2001 a 2009, sendo que, a principal foi dor torácica, seguida por dispnéia, dor abdominal e fraqueza ${ }^{11}$. Em outro, realizado no mesmo ano, $27 \%$ internaram por queixas inespecíficas, como astenia e náuseas, $13 \%$ por dispneia e $9 \%$ por febre ${ }^{12}$. Em 2014, o seguinte foi observado por estudo canadense: $9,1 \%$ por dispneia, $8,1 \%$ por dor torácica e $7,9 \%$ por dor em membros inferiores ${ }^{5}$. Estes achados são semelhantes ao encontrado na amostra.

As principais causas de internação foram infecção, e complicações de doenças oncológicas. Estes dados são alarmantes tendo em vista que as infecções contribuem com até um terço das mortes em pacientes com 65 anos ou mais ${ }^{13}$. Além disso, o reconhecimento desta condição representa um desafio, pois sinais típicos como febre e leucocitose podem estar ausentes ${ }^{13}$. Semelhante ao achado no estudo, outro trabalho ${ }^{5}$ apontou as neoplasias como importantes causas de admissão dos pacientes idosos nas unidades de emergência. Isso é coerente com a literatura que aponta que até $80 \%$ dos casos de câncer são diagnosticados em pacientes com 55 anos ou mais ${ }^{14}$.

Em um estudo nacional, doenças circulatórias e respiratórias foram responsáveis por, respectivamente, $17,6 \%$ e $8,2 \%$ dos atendimentos em idosos ${ }^{15}$. Estas condições comumente citadas na literatura ${ }^{16}$ como causas de apresentação do paciente idoso nos serviços de emergência tiveram, neste estudo, frequências menores que $10 \%$ cada. Outra observação é a falta de admissões por quedas, traumas ou acidentes, o que é frequentemente relatado na literatura ${ }^{5,8}$. Porém, é importante observar que no HCUFG, estes pacientes são direcionados ao ProntoSocorro da Ortopedia, cujos dados não foram incluídos na amostra do estudo.

A maior parte dos pacientes não apresentou complicações durante a internação, apesar de idosos terem maior chance de sofrerem declínio funcional e agravamentos, como quedas, úlceras de pressão e delirium nesse período ${ }^{17}$. Foi observado que apenas $9 \%$ das complicações durante a internação estiveram relacionadas com infecções, o que foi um valor abaixo do encontrado na literatura ${ }^{18}$.

Um estudo realizado em São Paulo observou que os eventos adversos durante a internação mais comuns eram os relacionados a drogas $(38.3 \%)$, seguidos de infecção hospitalar $(28,5 \%)$ e de procedimentos terapêuticos (16,8\%), como flebite em acesso venoso periférico. Os principais sítios acometidos por infecção hospitalar foram os pulmões $(29,5 \%)$ e o trato urinário $(19,6 \%)^{18}$, semelhante ao encontrado neste trabalho. Efeitos adversos relacionados a drogas ou procedimentos terapêuticos não foram relatados nos prontuários analisados.

As comorbidades encontradas nos pacientes estudados foram próximas aos resultados encontrados na literatura ${ }^{19}$. Uma revisão aponta que a presença de comorbidades em idosos varia de $55 \%$ a $98 \%$, e é maior em pacientes de classes econômicas mais desfavorecidas ${ }^{20}$, semelhante ao público que o Hospital das Clínicas assiste. As principais comorbidades encontradas foram consistentes com outros achados da literatura ${ }^{21}$, e com a transição epidemiológica que acompanha o aumento da expectativa de vida ${ }^{4}$. É importante ressaltar que a presença de várias condições crônicas está associada a uma pior qualidade de vida e declínio funcional, bem como à necessidade de tratamentos complexos e, por vezes, conflitantes ${ }^{22}$.

Além disso, observou-se que um percentual importante dos pacientes faz uso de 5 ou mais medicamentos continuamente. Os medicamentos considerados foram aqueles de uso crônico, não sendo possível obter informações sobre os que são usados sem a devida prescrição, como analgésicos e anti-inflamatórios. Nenhum paciente teve admissão na unidade de Pronto-Socorro por consequência de interações medicamentosas ou efeitos adversos de medicamentos, mas é demonstrado que até $10 \%$ das causas de internação em unidades de emergência, em pacientes com mais de 65 anos, pode decorrer das consequências da polifarmácia ${ }^{23}$. O uso de vários medicamentos também está associado a declínio funcional e outras complicações como quedas e deterioração cognitiva ${ }^{24}$.

A maior parte dos pacientes permaneceu quatro dias internada no Pronto-Socorro, sendo incluídos no estudo somente aqueles que ficaram mais de 24 horas no local. Isto difere da literatura consultada na qual essa média de tempo foi de 15,4 horas para pacientes admitidos e apenas $5 \%$ teriam permanecido mais de 24 horas no PS. A idade avançada foi um fator diretamente associado à estadia mais longa no hospital ${ }^{5}$. No HCUFG, a permanência prolongada no PS reflete um problema estrutural de falta de leitos para admissões em outras unidades, o que expõe esses idosos a maiores riscos de eventos adversos ${ }^{25}$.

A média de tempo de internação na UTI encontrada no estudo é discrepante em relação ao que foi encontrado em outro no qual os idosos que foram admitidos e liberados após melhora permaneceram na unidade por 7,03 dias, enquanto que os que vieram a óbito, por 10,15 $\operatorname{dias}^{25}$. Entretanto, não foi realizada análise ajustada por gravidade dos casos ou pelo número de óbitos, o que limita tais comparações. Apesar disso, outro trabalho mostra resultados mais próximos aos encontrados, com média de tempo de internação de 1,9 dia em UTI e evidências de que o número de dias no hospital era menor entre os pacientes que faleceram no local ${ }^{26}$.

Em quatro estudos internacionais consultados, as taxas de mortalidade foram: $0,02 \% ; 0,2 \%, 1,8 \%$ e $2,3 \%{ }^{6,7,27,28}$. Por outro lado, fazendo-se uma comparação em 
nível nacional, um estudo de Montes Claros registrou uma taxa de $12,7 \%$, quanto a evolução insatisfatória (quadro clínico inalterado ou evolução para óbito) em um período de 16 meses $^{29}$. Outro estudo prospectivo com tempo de seguimento de 9,4 anos teve $36 \%$ de mortalidade. Já no HCUFG, aproximadamente $18,47 \%$ complicaram e vieram a óbito, sendo este valor superior ao encontrado em relação à literatura internacional, porém semelhante aos achados nacionais.

Observando-se esta taxa de mortalidade alarmante, especialmente se comparada à literatura internacional acima citada, é possível questionar se a mesma é um reflexo da dificuldade de acesso do paciente ao serviço de saúde, já que este é regulado somente quando tem a vaga disponível. Outras hipóteses seriam: indisponibilidade de recursos materiais e humanos e o menor acesso à tecnologia médica de diagnóstico e tratamento; perfil mais grave dos pacientes com doenças em estágio avançado e múltiplas complicações; baixa condição social e educacional da população atendida.

Um fato importante a ser mencionado é a limitação do trabalho quanto à classificação de risco dos pacientes na triagem. A classificação de risco de Manchester ${ }^{30}$, foi implementada no HCUFG no segundo semestre de 2015, de maneira que grande parte dos pacientes que chegaram ao PS não foi estratificada de forma padronizada. Isto dificultou o estabelecimento da relação entre gravidade na triagem, complicações e óbitos durante a internação, assim como a comparação dos dados com a literatura internacional.

Além disso, por se tratar de um estudo com coleta de dados dos prontuários, é importante ressaltar as limitações e dificuldades inerentes à fonte de dados. O preenchimento manual dos profissionais assistencialistas está sujeito a erros de registro ou má qualidade. Espera-se que por meio da padronização da coleta de dados, possíveis vieses tenham sido minimizados.

\section{CONCLUSÃO}

Conclui-se que o perfil de idosos atendidos no PSHCUFG consiste em pacientes principalmente do sexo masculino, pardos e com mediana de idade de 69 anos. A maioria apresentou queixas de náuseas e vômitos na triagem e infecção foi a principal causa de internação. Houve um predomínio de pacientes com pelo menos 3 comorbidades, sendo diabetes mellitus e hipertensão arterial as mais importantes. Boa parte apresentou polifarmácia. Em geral, evoluíram sem complicações e receberam alta com melhora do quadro, embora o número de óbitos tenha sido importante no período.

Os achados mostram-se coerentes em grande parte com a literatura comparada. Espera-se que, através destes dados, seja possível contribuir com os estudos nacionais sobre pacientes idosos atendidos em serviços médicos de emergência, tendo em vista a necessidade crescente do desenvolvimento de cuidados de saúde voltados para esse grupo.

O olhar sobre o idoso deve ser amplo e cuidadoso. Os médicos e outros profissionais que assistem à saúde em unidades de emergência costumam não ter experiência específica sobre como lidar com esta faixa etária. Sendo assim, mais estudos como o apresentado são necessários para criar uma base sólida de conhecimentos que direcionem abordagens mais resolutivas e eficazes.

Participção dos autores: Cacilda Pedrosa de Oliveira - Idealização do projeto, participando da discussão para definição dos critérios pré-projeto, participação da construção do formulário a ser utilizado, orientação da capacitação da equipe, oferecendo insumos teóricos e experiência prática, participação da revisão da análise crítica dos dados, bem como, auxílio na definição dos fatores mais importantes, coordenação da equipe de execução do projeto, revisão e opinião crítica acerca do texto final da projeto; Andrey Rocha Rocca - Idealização do projeto, participando da discussão para definição dos critérios pré-projeto, participação da construção do formulário a ser utilizado, coordenação da execução da coleta de dados, coleta de dados dos prontuários, tabulação de dados em ambiente informatizado, realização de análise estatística, escrita de parte documento final, baseado em análise e revisão de literatura, revisão do documento final; Isadora Marques Guimarães Santos - Idealização do projeto, participando da discussão para definição dos critérios pré-projeto, participação da construção do formulário a ser utilizado, coordenação da execução da coleta de dados, coleta de dados dos prontuários, tabulação de dados em ambiente informatizado, realização de revisão de literatura para oferecer base teórica ao projeto, escrita de parte documento final, baseado em análise e revisão de literatura, revisão do documento final; Gabriel Duarte do Nascimento - Participação das definições práticas do projeto, bem como as reuniões de aprimoramento do projeto inicial, coleta de dados dos prontuários, tabulação de dados em ambiente informatizado, realização de revisão de literatura para oferecer base teórica ao projeto, escrita de parte documento final, baseado em análise e revisão de literatura; Giovani Prediger Dobri - Participação das definições práticas do projeto, bem como as reuniões de aprimoramento do projeto inicial, coleta de dados dos prontuários, tabulação de dados em ambiente informatizado, construção dos quadros utilizados no artigo, realização de revisão de literatura para oferecer base teórica ao projeto, escrita de parte documento final, baseado em análise e revisão de literatura.

\section{REFERÊNCIAS}

1. Beard JR, Officer AM, Cassels AK. The world report on ageing and health. Gerontologist. 2016;56:S163-6. doi: 10.1093/geront/gnw037.

2. Vasconcelos AMN, Gomes MMF. Transição demográfica: a experiência brasileira. Epidemiol Serviços Saúde. 2012;21(4):539-48. doi: 10.5123/S167949742012000400003.
3. Bezerra FC, Almeida MI De, Nóbrega-Therrien SM. Estudos sobre envelhecimento no Brasil: revisão bibliográfica. Rev Bras Geriatr Gerontol. 2012;15(1):155-67. doi: 10.1590/ S1809-98232012000100017.

4. World Health Organization. National Institute on Aging, National Institutes of Health, U.S. Department of Health and Human Services. Global health and aging. Geneva; 2011. 
(NIH Publ no 11-7737). Available from: http://www.who. int/ageing/publications/global_health.pdf.

5. Latham LP, Ackroyd-Stolarz S. Emergency department utilization by older adults: a descriptive study. Can Geriatr J. 2014;17(4):118-25. doi: 10.5770/cgj.17.108.

6. Salvi F, Morichi V, Grilli A, Giorgi R, De Tommaso G, Dessi-Fulgheri P. The elderly in the emergency department: a critical review of problems and solutions. Intern Emerg Med. 2007;2(4):292-301. doi: http://dx.doi.org/10.1007/ s11739-007-0081-3.

7. McCabe JJ, Kennelly SP. Acute care of older patients in the emergency department: strategies to improve patient outcomes. Open Access Emerg Med. 2015;7:45-54. doi: 10.2147/OAEM.S69974.

8. Samaras N, Chevalley T, Samaras D, Gold G. Older patients in the emergency department: a review. Ann Emerg Med. 2010;56(3):261-9. doi: http://dx.doi.org/10.1016/j. annemergmed.2010.04.015.

9. Quinn K, Herman M, Lin D, Supapol W, Worster A. Common diagnoses and outcomes in elderly patients who present to the emergency department with non-specific complaints. Can J Emerg Med. 2015;17(5):516-22. doi: http://dx.doi.org/10.1017/cem.2015.35.

10. U.S. Department of Health and Human Services, Centers for Disease Control and Prevention, National Center for Health Statistics. National Hospital Ambulatory Medical Care Survey: 2012 emergency department summary tables. United States: Centers for Disease Control and Prevention; 2013. Available from: http://www.cdc.gov/nchs/data/ahcd/ nhamcs_emergency/2012_ed_web_tables.pdf.

11. Pines JM, Mullins PM, Cooper JK, Feng LB, Roth KE. National trends in emergency department use, care patterns, and quality of care of older adults in the United States. J Am Geriatr Soc. 2013;61(1):12-7. doi: 10.1111/jgs. 12072.

12. Fayyaz J, Khursheed M, Umer M, Khan U. Pattern of emergency department visits by elderly patients: study from a tertiary care hospital, Karachi. BMC Geriatr. 2013;13:83. doi: http://dx.doi.org/10.1186/1471-2318-13-83.

13. Mouton CP, Bazaldua O V., Pierce B, Espino D V. Common infections in older adults. Am Fam Phys. 2001;63:257-68. Available from: http://familymed.uthscsa.edu/geriatrics/ reading $\% 20$ resources/virtual_library/ID/Common $\% 20$ Infections01.pdf.

14. Marosi C, Koller M. Challenge of cancer in the elderly. ESMO Open. 2016;3(1):1-6. doi: http://doi.org/10.1136/ esmoopen-2015-000020.

15. Stein FC, Barros RK, Feitosa FS, Toledo DO, da Silva Junior $\mathrm{JM}$, Ísola AM, et al. Fatores prognósticos em pacientes idosos admitidos em unidade de terapia intensiva. Rev Bras Ter Intensiva. 2009;21(3):255-61. doi: 10.1590/S0103$507 X 2009000300004$.

16. Canadian Institute for Health Information. Seniors' use of emergency departments in Ontario, 2004-2005 to 20082009. Ottawa, Ont.: CIHI; 2010. Available from: https:// secure.cihi.ca/free products/seniors ed e.pdf.

17. Fox MT, Persaud M, Maimets I, O'Brien K, Brooks D, Tregunno D, et al. Effectiveness of acute geriatric unit care using acute care for elders components: A systematic review and meta-analysis. Vol. 60, J Am Geriatr Soc. 2012;60:2237-45. doi: http://dx.doi.org/10.1111/jgs.12028.

18. Szlejf C. Eventos adversos médicos em idosos hospitalizados : frequência e fatores de risco em enfermaria de geriatria [tese]. São Paulo: Faculdade de Medicina, Universidade de São Paulo; 2010. doi: 10.11606/T.5.2010. tde-30112010-152956.

19. Schrijver EJM, Toppinga Q, de Vries OJ, Kramer MHH, Nanayakkara PWB. An observational cohort study on geriatric patient profile in an emergency department in the Netherlands. Neth J Med. 2013;71(6):324-30. Available from: http://www.njmonline.nl/getpdf.php?id=1337.

20. Nobili A, Garattini S, Mannucci P. Multiple diseases and polypharmacy in the elderly: challenges for the internist of the third millennium. J Comorbidity. 2011;1(1):28-44. doi: 10.15256/joc.2011.1.4.

21. Piccirillo JF, Vlahiotis A, Barrett LB, Flood KL, Spitznagel EL, Steyerberg EW. The changing prevalence of comorbidity across the age spectrum. Crit Rey Oncol Hematol. 2008;67(2):124-32. doi: http://dx.doi. org/10.1016/j.critrevonc.2008.01.013.

22. Caughey G. Multiple chronic health conditions in older people: implications for health policy planning, practitioners, and patients. Australia: University of South Australia, University of Adelaide, Flinders University; 2013. Available from: https://www.unisa.edu.au/Global/ Health/Sansom/Documents/QUMPRC/Multiple-ChronicHealth-Conditions.pdf.

23. Graham E, Marshall T, Ritchie C. Comprehensive geriatric assessment in the emergency department. Clin Interv Aging. 2014;9:2033-43. doi: http://dx.doi.org/10.2147/CIA S29662.

24. Maher RL, Hanlon J, Hajjar ER. Clinical consequences of polypharmacy en elderly. J Expert Opin Drug Safety. 2014;13(1):57-65. doi: http://dx.doi.org/10.1517/1474033 8.2013 .827660

25. Said SMS El. Geriatrics intensive care unit: outcome and risk factors for in hospital mortality. Adv Aging Res. 2013;2(4):166-9. doi: http://dx.doi.org/10.4236/ aar.2013.24024.

26. Duke GJ, Barker A, Knott CI, Santamaria JD. Outcomes of older people receiving intensive care in Victoria. Med J Aust. 2014;200(6):323-6. doi: http://dx.doi.org/10.5694/ mja13.10132.

27. Keskinoglu P, Inan F. Analysis of emergency department visits by elderly patients in an urban public hospital in Turkey. J Clin Gerontol Geriatr. 2014;5(4):127-31. doi: 10.1016/j.jcgg.2014.07.001.

28. Hu SC, Yen D, Yu YC, Kao WF, Wang LM. Elderly use of the ED in an asian metropolis. Am J Emerg Med. 1999;17(1):95-9. doi: http://dx.doi.org/10.1016/s07356757(99)90029-3.

29. Rabelo LP de O, Vieira MA, Caldeira AP, Costa S de M. Perfil de idosos internados em um hospital universitário. REME, Rev Min Enferm. 2010;14(3):293-300. Disponível em: http://www.revenf.bvs.br/scielo.php?script=sci arttext\&pid=S1415-27622010000300002\&lng=es\&nrm= iso\&tlng $=\mathrm{pt}$.

30. de Souza CC, Araújo FA, Chianca TCM. Scientific literature on the reliability and validity of the manchester triage system (MTS) protocol: a integrative literature review. Rev Esc Enferm USP. 2015;49(1):144-51. doi: http://dx.doi. org/10.1590/s0080-623420150000100019.

Recebido em: 05.12.17

Aceito em: 09.01.18 